\title{
Evaluation for Vertebral Artery Injury with originall Cervical Dislocated Fracture and Optimal Treatment before Reduction
}

Yasuo Suga, ,1,2 Yumiko Mitome-Mishima, ,,2 Kensaku Yoshida, ${ }^{1,2}$ Takuma Higo, 1,2 Kazuki Nishioka, 1,2 and Hidenori Oishi',3

Objective: Cervical dislocated fractures frequently cause vertebral artery injury (VAI), which, in turn, propagates the thrombus at the site of injury. Cerebral embolism due to a thrombus after the reduction of dislocation leads to a poorer neurological outcome. Therefore, we investigated the outcome of treatment for cervical dislocated fractures and the usefulness of parent artery occlusion (PAO) before reduction.

Methods: Eight patients with cervical dislocated fractures with a locked facets treated at our hospital between January 2018 and December 2020 were evaluated. We retrospectively examined patient characteristics and clinical outcomes.

Results: Among the eight patients, two were injured at $\mathrm{C} 4 / 5$, four at $\mathrm{C} 5 / 6$, and two at $\mathrm{C} 6 / 7$. All patients had locked facets. Four patients had bilateral dislocation, while the others had unilateral dislocation. Two patients with unilateral dislocation had ipsilateral vertebral artery occlusion (VAO), while the other six did not. Both patients with VAO underwent PAO to prevent cerebral embolism before reduction. The six patients who did not have VAI underwent reduction without preprocedural treatment. No cerebral ischemic complications were observed. One patient died due to paralysis of the respiratory muscles caused by spinal cord injury but the remaining seven recovered well.

Conclusion: PAO before reduction for cervical dislocated fractures with VAO may be effective in preventing cerebral embolism after reduction.

Keywords > cervical dislocated fracture, vertebral artery injury, parent artery occlusion

\section{Introduction}

Research shows that the rate of cervical spine injury complicated by vertebral artery injury (VAI) is approximately $19 \%-47 \%$, with the rate of complication by VAI-induced cerebral infarction at approximately $0 \%-54 \%$, and the

${ }^{1}$ Department of Neurosurgery, Tokyo Metropolitan Hiroo Hospital, Tokyo, Japan

${ }^{2}$ Department of Neurosurgery, Juntendo University School of Medicine, Tokyo, Japan

${ }^{3}$ Department of Neuroendovascular Therapy, Juntendo University School of Medicine, Tokyo, Japan

Received: March 31, 2021; Accepted: July 17, 2021

Corresponding author: Yasuo Suga. Department of Neurosurgery, Tokyo Metropolitan Hiroo Hospital, 2-34-10, Ebisu, Shibuya-ku, Tokyo 150-0013, Japan

Email: yssuga@juntendo.ac.jp

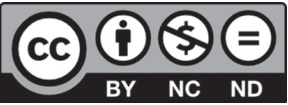

This work is licensed under a Creative Commons Attribution-NonCommercialNoDerivatives International License.

(C)2021 The Japanese Society for Neuroendovascular Therapy mortality at approximately $0 \%-18 \% .^{1-4)}$ Moreover, $7 \%-20 \%$ of cervical spine injury patients develop vertebral artery occlusion (VAO) as a result of VAI.,5) On the contrary, cervical spine fracture is noted in $70 \%$ or more VAI cases. ${ }^{6}$ VAI complication rate with facet dislocation, subluxation, and fracture of the transverse foramen were reported in $80 \%, 75 \%$, and $46 \%$ of cases, respectively. ${ }^{1,3,7-9)}$ However, for treatment, reports of course observation, antithrombotic drug administration, and parent artery occlusion (PAO) of the affected vertebral artery are mixed with no consensus, as the pathology and complicating injury differ among patients. ${ }^{1,2,9,10)}$ In patients requiring reduction of dislocation and fusion, there is a risk of fatal cerebral embolism due to scattering of the thrombus formed in the VAI lesion after restoration of the vertebral artery by reduction. ${ }^{11,12)}$ Thus, we retrospectively investigated the treatment outcome of cervical dislocated fractures that required reduction of dislocation and the efficacy of endovascular PAO applied before reduction of dislocation in our patients. We report this study with a review of the literature. 


\section{Materials and Methods}

\section{Subjects}

The subjects were patients with cervical dislocated fractures who required a reduction in dislocation and presented to our hospital between January 2018 and December 2020 . Those with neurological manifestations that were difficult to assess due to disturbance of consciousness were excluded.

The patient characteristics and other pertinent history, such as the age, sex, treatment performed before reduction of dislocation, mechanism of injury, region injured by dislocation fracture, dominance of the vertebral artery, presence of VAI and cerebral embolism before and after reduction of dislocation, surgical procedure for spinal fusion, American Spinal Cord Association impairment scale on admission and at discharge, and modified Rankin Scale (mRS) score at discharge were investigated.

\section{Neuroradiological diagnosis}

On admission, plain CT of the head and cervical spine as well as spinal cord MRI was performed to evaluate intracranial and spinal or spinal cord injuries. CTA was employed as the first choice for VAI before reduction of dislocation. After reduction, MRA was employed as the first choice, and when a change was noted compared with CTA or MRA before reduction, CTA was performed additionally. Diffusion-weighted head MRI was performed before and after the reduction for comparison.

\section{Endovascular treatment before reduction of dislocation}

Of the patients diagnosed with VAO on neuroradiological examination, only those without cerebral ischemia were treated with PAO to prevent subsequent cerebral embolism. The balloon occlusion test (BOT) was omitted because the vessels were occluded. 5-F long sheath (Medikit, Tokyo, Japan) was inserted into the right common femoral artery under local anesthesia, and diagnostic angiography was performed to evaluate collaterals. 5-F Envoy MPC (Johnson \& Johnson, New Brunswick, NJ, USA) was introduced to the proximal part of the injured vertebral artery thereafter. Systemic heparinization was not performed. Thereafter, an Excelsior 1018 (Stryker, Kalamazoo, MI, USA) was introduced into the lesion, and the injured vertebral artery was occluded with platinum coils. No antithrombotic therapy was administered postoperatively.

\section{Ethics guidelines for research}

This study was approved by the Research Ethics Committee for Clinical Studies of our hospital and was performed in accordance with the 1964 Declaration of Helsinki and ethical codes established in later revisions.

\section{Results}

The subjects were eight patients, and none of them met the exclusion criteria. All patients are presented in Table 1. There were five males and three females, and the mean age was $56.5( \pm 20.1)$ years. The mechanism of injury was overflexion of the neck in seven patients, and the dislocated sites were $\mathrm{C} 4 / 5$ and $\mathrm{C} 6 / 7$ in two patients, and C5/6 in four. There were four unilateral and bilateral cases each, and all were accompanied by a locked facet. Ipsilateral VAO was noted in unilateral cases of $\mathrm{C} 4 / 5$ and $\mathrm{C} 5 / 6$ dislocation, while no VAI was noted in the other cases. No cerebral neurological manifestations were observed. Endovascular PAO of the affected blood vessel was performed before the reduction of dislocation in two patients complicated by VAO, whereas reduction was performed without treatment in the other patients. Of the patients treated using PAO, case 1 was of a lesion of the vertebral artery on the non-dominant side and case 2 was of a lesion on the dominant side. The time required to complete PAO from injury was 15 hours and 50 minutes in case 1 and 6 hours and 40 minutes in case 2 . The interval between the completion of PAO and the initiation of dislocation reduction was 2 hours in case 1 and 1 hour and 20 minutes in case 2. No antithrombotic drugs were administered to any patient throughout the study. No cerebral ischemic symptoms or novel ischemic lesions on head MRI developed after reduction in any patient. No new VAI was noted in any patient on cervical MRA or CTA after reduction. Subsequently, spinal fusion was performed in all patients. One patient died of spinal cord injury-derived paralysis of the respiratory muscles, but a favorable outcome was observed in seven patients.

\section{Representative case}

Case 2

A 60-year-old man fell and was injured by neck overflexion. There was no cerebral neuropathy at the time of arrival but left-dominant quadriparesis and dysfunction of sensation in the left upper limb and bilateral lower limbs were observed. On cervical spine CT and neck CTA, a dislocation fracture accompanied by locked facet of the 


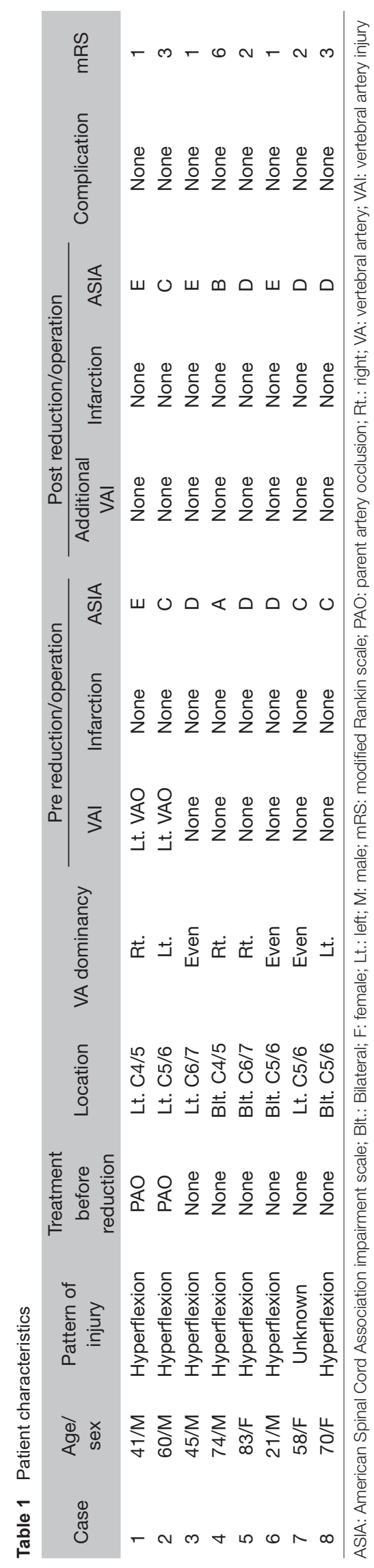

left $\mathrm{C} 5 / 6$ and VAO of the same region were noted. However, visualization of the vertebral artery distal to the occluded region was favorable, suggesting the involvement of collateral circulation. The contralateral right vertebral artery distal to the posterior inferior cerebellar artery bifurcation was hypoplastic. Angiography revealed collateral flow from the deep cervical branch of the costocervical trunk and muscular branch of the occipital artery to the left vertebral artery distal to the occluded region; antegrade perfusion of the right vertebral artery, albeit hypoplastic, to the basilar artery was also observed. As perfusion of the intracranial posterior circulation was maintained and cerebral ischemic symptoms were absent, PAO of the left vertebral artery was performed to prevent cerebral embolism (Fig. 1). Subsequently, the reduction of dislocation and anterior decompression and fusion were performed in the orthopedic department. None of these treatments caused complications, and the patient was transferred with an $\mathrm{mRS}$ score of 3 to another hospital for rehabilitation.

\section{Discussion}

VAI frequently develops in the V2 segment, which is fixed by the transverse foramen and receives a strong shear force. It is usually caused by the overextension of the vertebral artery between the adjacent transverse foramina due to overflexion or overextension of the neck. ${ }^{5,6}$ ) There is no consensus concerning which intervertebral segment is likely to cause complications by VAI, ${ }^{3,6,11)}$ but among our patients, no VAI was observed in two $\mathrm{C} 6 / 7$ cases, which may have been due to high mobility of the vertebral artery and high interferential action for shear stress at C6/7 compared with the other intervertebral segments. Although we were unable to find reports on the VAI complication rates of unilateral and bilateral dislocation in our patients, it was noted in two of four patients with unilateral dislocation, suggesting an influence of torsion of the vertebral artery.

Regarding the pathology of VAI, many studies have reported that the essential condition is arterial wall injury by the fracture fragment and dissection accompanying arterial wall extension. ${ }^{5,6,13)}$ The neurological outcome is aggravated by cerebral embolism due to thrombus formation in the injured tunica intima during the course of injury, or hemodynamic cerebral ischemia accompanying stenosis or occlusion of the region caused by mural thrombus., ${ }^{313)}$ In addition, vascular rupture-induced false 

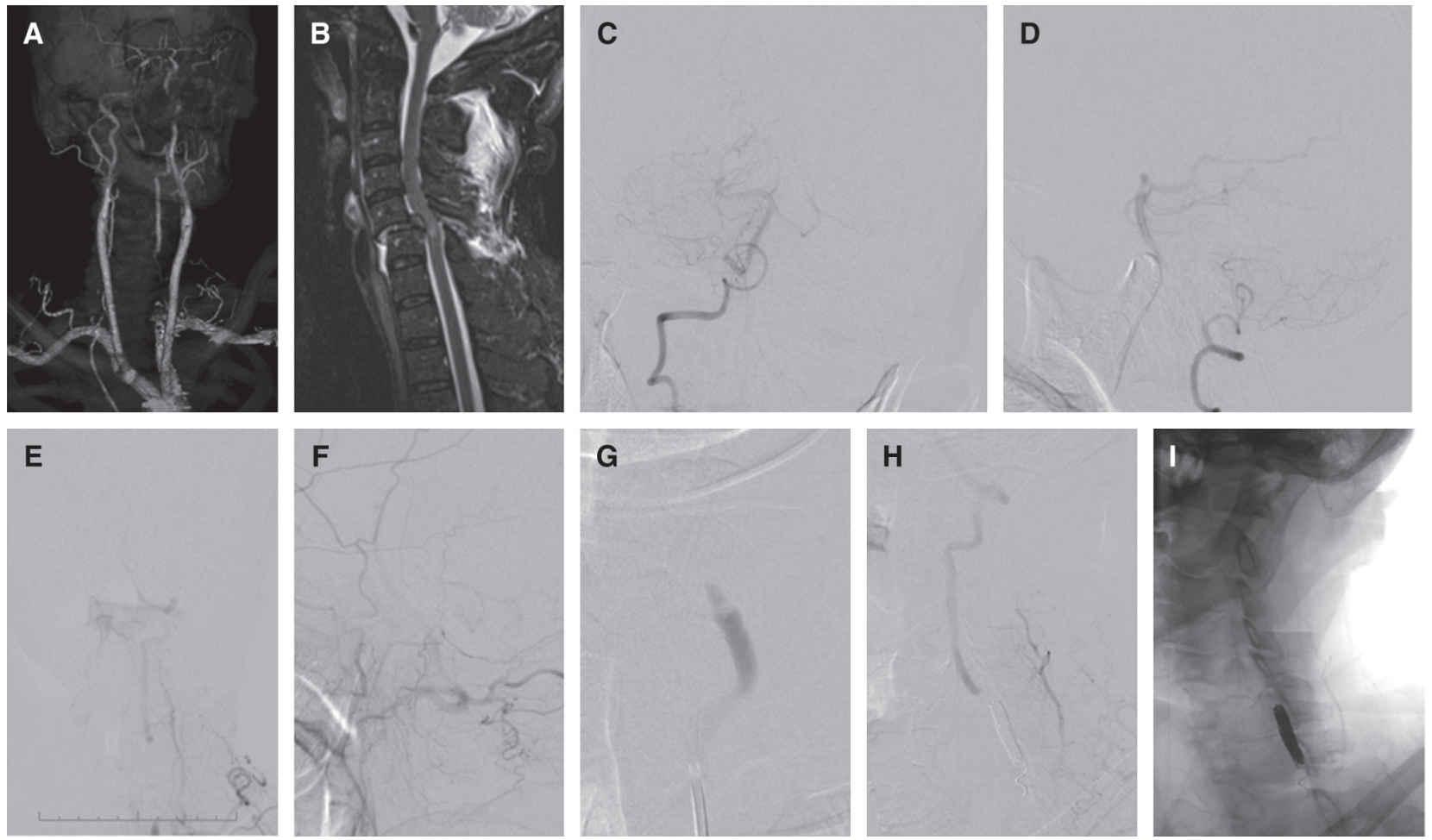

Fig. 1 (A) Right anterolateral view on 3D CTA on admission reveals left VAO. (B) Sagittal T2-weighted MRI shows spinal cord injury at C5/6. (C and D) Posterior-anterior and lateral views on angiography of the right vertebral artery show hypoplasty in the distal portion. ( $E$ and $\mathbf{F}$ ) The deep cervical artery from the costocervical trunk and the muscular branch of the occipital artery anastomosed to the

aneurysms and arteriovenous fistulas have been reported. ${ }^{11,13)}$ Among our patients, VAO was noted in two and PAO was performed before the reduction of dislocation, whereas dislocation was reduced without treatment in the other six patients because no VAI was noted and favorable outcomes were acquired for all. Thus, we further reviewed reports of PAO for cervical dislocated fractures complicated by VAO before the reduction of dislocation.

First, regarding factors involved in the manifestation of VAO symptoms, the degree of development of collateral circulation, dominance of the vertebral artery, arteriosclerosis, and aging have been reported.7) Many patients with unilateral VAO are asymptomatic because of collateral circulation, while bilateral VAO results in serious sequelae or death in many cases. ${ }^{5)}$ However, even unilateral development causes serious sequelae or death when a scattering thrombus causes basilar artery occlusion (BAO). ${ }^{7,8,9,12)}$ On the contrary, even in bilateral VAO, when BAO is absent, symptoms are mild because of collateral circulation in rare $\operatorname{cases}^{5,8)}$; therefore, avoiding embolism of the cerebral major arteries, including $\mathrm{BAO}$, may be the most important factor for the reduction of dislocation in patients with VAO. vertebral artery. (G) Contrast injection from the guiding catheter before PAO reveals complete occlusion of the left vertebral artery at the site of VAI. ( $\mathbf{H}$ and I) Left oblique view on angiography of the left subclavian artery after PAO. PAO: parent artery occlusion; VAl: vertebral artery injury; VAO: vertebral artery occlusion

Thus, we searched for reports on treatment before the reduction of dislocation in patients with VAO. There is a method for performing reduction under antithrombotic drug administration and another using endovascular treatment after PAO, but these are controversial at present.

Regarding the reduction of dislocation under antithrombotic drug administration, it was reported to be safe for asymptomatic patients ${ }^{14)}$ and cerebral embolism after reduction was avoided using aspirin administration. ${ }^{9)}$ In addition, early initiation of anticoagulant therapy, reduction of dislocation, and spinal fusion were reported to be effective in inhibiting thrombus formation and preventing cerebral embolism. ${ }^{4,68)}$ However, death due to reduction accompanied by $\mathrm{BAO}^{12}$ and the development of multiple cerebral embolisms ${ }^{10}$ ) have been reported, leaving questions about safety. preceded by PAO using endovascular treatment, no complications were noted after PAO or the reduction of dislocation in previously reported cases. ${ }^{2,3,11)}$ Indo et al. ${ }^{11)}$ retrospectively investigated 23 patients with cervical spine fractures complicated by VAO that required spinal fusion in the acute
On the contrary, regarding the reduction of dislocation 
phase. Cerebral infarction was identified after surgery in five of nine patients not treated with PAO before surgery, whereas it was absent in all 14 patients treated using PAO before surgery, and only preoperative PAO was associated with the prevention of postoperative cerebral infarction on univariate analysis. When only 19 patients treated by reduction were extracted, a similar trend was noted; cerebral infarction was noted after surgery in five of the nine patients not treated using PAO, but it was not noted in any of the 10 patients treated using PAO, suggesting that the risk of PAO embolizing an already occluded blood vessel is low and the cerebral embolism-preventive effects are high in VAO patients requiring reduction of dislocation, which suggests the suitability of the treatment method. ${ }^{11)}$

Regarding BOT before PAO, it has been reported that when occlusion or severe stenosis is noted on admission, the patient can be judged as tolerant to functional occlusion testing at this time point, making BOT unnecessary. ${ }^{15)}$ Among our patients, PAO was performed on patients with $\mathrm{VAO}$ on the dominant and non-dominant sides, and favorable outcomes were acquired, consistent with the above conclusion.

Regarding the region embolized by PAO, only the proximal region was occluded in some reports, ${ }^{3,11}$ and both proximal and distal regions were occluded in others, with internal trapping. ${ }^{2)}$ We performed PAO using the former method and found no cerebral embolism after the reduction of dislocation. We performed PAO on only the proximal region of the injury because first, it should be completed quickly because it is positioned as a pretreatment for the reduction of dislocation, which should be performed as rapidly as possible. Second, to perform trapping, an approach through the contralateral vertebral artery is necessary, with which the complications described below may occur. Specifically, the aggravation of contralateral VAI that may be latently present, iatrogenic contralateral VAI that may be fatal, and the reduction of basilar artery perfusion due to contralateral catheterization with occlusion of the affected side and accompanying ischemic complications are considered. On the contrary, researchers adopting internal trapping unlike ours may have concerns over the development of pathology similar to the vertebral artery stump syndrome after PAO of only the proximal region. ${ }^{16-18)}$ The vertebral artery stump syndrome represents cerebral embolism caused by intracranial transport of the thrombus formed around the distal stump of the region occluded by antegrade collateral flow from the subclavian artery branch in patients with chronic arteriosclerotic occlusion of the origin of the vertebral artery; that is, in internal trapping, PAO is applied at the distal junction at which antegrade collateral flow from the subclavian artery branch joins the vertebral artery. However, unlike chronic occlusion of the origin of the vertebral artery, the stump syndrome may be less likely to occur immediately after PAO with poor collateral circulation to the cervical vertebral artery distal to occlusion. Even if the stump syndrome occurs, the possibility of embolism by a large thrombus causing occlusion of the cerebral major arteries may be low. By comprehensively considering the characteristics of each of these treatments and the main purpose of avoiding embolism of the major cerebral arteries by reduction of dislocation, we performed PAO of the proximal region of the injury as the first choice as it can be performed simply, safely, and quickly.

There are some limitations to this study. First, it was a single-center retrospective study involving a small number of patients. Second, no patients with VAI other than VAO were included, and the long-term outcome is unclear. Therefore, it is necessary to perform an organized randomized controlled trial in addition to increasing the sample size in the future.

\section{Conclusion}

When reduction of dislocation is applied to cervical dislocated fractures, endovascular PAO before reduction may be effective in preventing cerebral embolism after reduction in patients with $\mathrm{VAO}$; therefore, performing this procedure may be considered. However, a large sample size and long-term follow-up of the outcomes may be necessary.

\section{Acknowledgment}

We would like to thank Editage (www.editage.com) for English language editing.

\section{Disclosure Statement}

The authors declare no conflicts of interest.

\section{References}

1) Fujita $Y$, Aihara $H$, Nagashima $H$, et al. [Clinical features and treatment strategy of vertebral artery injury associated with cervical spine trauma]. No Shinkei Geka 2018; 46: 663-671. (in Japanese) 
2) Zou $\mathrm{HJ}, \mathrm{Wu} J, \mathrm{Hu} \mathrm{Y}$, et al. Unilateral vertebral artery injury in a patient with displaced upper cervical spine fractures: the treatment for one case of vertebral artery embolism. Eur Spine J 2018; 27: 409-414.

3) Isaji T, Ohshima T, Nakura T, et al. Efficacy of endovascular proximal occlusion before direct reposition surgery of blunt cervical fracture with unilateral vertebral injury. NMC Case Report J 2019; 6: 131-134.

4) Foreman PM, Griessenauer CJ, Chua M, et al. Corrective spinal surgery may be protective against stroke in patients with blunt traumatic vertebral artery occlusion. $J$ Neurosurg Spine 2015; 23: 665-670.

5) Strickland B, Lewis CS, Pham MH. Bilateral vertebral artery occlusion after cervical spine fracture dislocation. World Neurosurg 2019; 124: 304-309.

6) Yaeger K, Mascitelli J, Kellner C, et al. Temporary vertebral artery occlusion after $\mathrm{C} 3$ fracture dislocation injury and spontaneous resolution following reduction and instrumented fusion: case report and literature review. J Neurointerv Surg 2017; 9: 1027-1030.

7) Shibata $T$, Ogiichi $T$, Miyake $T$, et al. [A case of basilar artery occlusion of traumatic vertebral artery dissection successfully managed by endovascular treatment]. No Shinkei Geka 2003; 31: 311-316 (in Japanese).

8) Davies SR. A rare complication of a unilateral vertebral artery occlusion, which resulted in a basilar emboli after a C5-C6 bifacet dislocation in a professional rugby player: case study. Spine J 2011; 11: e1-e4.

9) Sack JA, Etame AB, Shah GV, et al. Management and outcomes of patients undergoing surgery for traumatic cervical fracture-subluxation associated with an asymptomatic vertebral artery injury. J Spinal Disord Tech 2009; 22: 86-90.

10) Nakao Y, Terai H. Embolic brain infarction related to posttraumatic occlusion of vertebral artery resulting from cervical spine injury: a case report. J Med Case Rep 2014; 8: 344.

11) Indo M, Oya S, Shojima M, et al. Prevention of thromboembolic infarction after surgery for traumatic cervical fracture with vertebral artery occlusion by preoperative endovascular coil embolization. World Neurosurg 2019; 129: e838-e844.

12) Tumialán LM, Theodore N. Basilar artery thrombosis after reduction of cervical spondyloptosis: a cautionary report. J Neurosurg Spine 2012; 16: 492-496.

13) Nagpal P, Policeni BA, Bathla G, et al. Blunt cerebrovascular injuries: advances in screening, imaging, and management trends. AJNR Am J Neuroradiol 2018; 39: 406-414.

14) Willis BK, Greiner F, Orrison WW, et al. The incidence of vertebral artery injury after midcervical spine fracture or subluxation. Neurosurgery 1994; 34: 435-442.

15) Zoarski GH, Seth R. Safety of unilateral endovascular occlusion of the cervical segment of the vertebral artery without antecedent balloon test occlusion. AJNR Am J Neuroradiol 2014; 35: 856-861.

16) Suzuki M, Dembo T, Hara W, et al. Vertebral artery stump syndrome. Intern Med 2018; 57: 733-736.

17) Nguyen TN, Raymond J, Mahmoud M, et al. Vertebral artery stump syndrome. J Neurol Neurosurg Psychiatry 2008; 79: 91-92.

18) Kawano $H$, Inatomi $Y$, Hirano $T$, et al. Vertebral artery stump syndrome in acute ischemic stroke. $J$ Neurol Sci 2013; 324: 74-79. 